\title{
Derivation of System Matrix From Simulation Data for an Animal SPECT With Slit-Slat Collimator
}

\author{
Rutao Yao, Member, IEEE, Tianyu Ma, Member, IEEE, and Yiping Shao, Member, IEEE
}

\begin{abstract}
We developed SPECT imaging capability on an animal PET system. Our goal was to provide animal PET users the SPECT capability at a low cost and facilitate potential PET/SPECT dual modality imaging applications. The SPECT function was enabled with a slit-slat collimator insert and by acquiring data in singles mode. The focus of this paper is to establish a method for deriving the system matrix for the SPECT system from Monte Carlo simulation. With the Monte Carlo package GATE, we simulated a uniform cylinder source which filled the SPECT field of view (FOV). To reduce the size of the original large and sparse system matrix, the detectors that were exposed to individual emission elements were selectively included for system matrix derivation and storage. The axial symmetry of the system was exploited so that only the base-axial volume was used for deriving system response. The system matrix derived was validated with point source measurements at known positions and implemented in an iterative reconstruction algorithm. The imaging performance of the system matrix was evaluated with experimental phantom studies. Reconstructed phantom images were artifact free and demonstrated expected spatial resolution. The method presented in this work is generally applicable to other SPECT imaging systems.
\end{abstract}

Index Terms-Image reconstruction, Monte Carlo simulation, PET/SPECT dual tracer imaging, SPECT, system matrix.

\section{INTRODUCTION}

$\mathbf{T}$ O expand the availability of SPECT for biomedical research, we studied the feasibility of adding a collimator to an existing animal PET scanner to make a "converted" SPECT. The benefits of this concept include 1) lower overall cost for having both PET and SPECT capability; 2) less space requirements as compared to having two separate systems; and 3) intrinsic PET and SPECT registration for potential PET/SPECT dual tracer research protocols. We selected the slit-slat collimator [1]-[2] for the prototype system [3], to maximize the use of the PET ring detectors, and to benefit from the uniform axial

Manuscript received September 18, 2008; revised February 03, 2009. Current version published October 07, 2009. This work was supported in part by Roswell Park Alliance Foundation Grant 62-2426-01.

R. Yao is with the Department of Nuclear Medicine, State University of New York at Buffalo, Buffalo, NY 14214 USA (e-mail: rutaoyao@buffalo.edu).

T. Ma is with the Department of Nuclear Medicine, State University of New York at Buffalo, Buffalo, NY 14214 USA and also with the Department of Engineering Physics, Tsinghua University, Beijing, China (e-mail: tianyuma@buffalo.edu).

Y. Shao was with the Department of Nuclear Medicine, State University of New York at Buffalo, Buffalo, NY 14214 USA. He is now with the Department of Imaging Physics, University of Texas M.D. Anderson Cancer Center, Houston, TX 77030 USA (e-mail: Yiping.Shao@di.mdacc.tmc.edu).

Color versions of one or more of the figures in this paper are available online at http://ieeexplore.ieee.org.

Digital Object Identifier 10.1109/TNS.2009.2022940

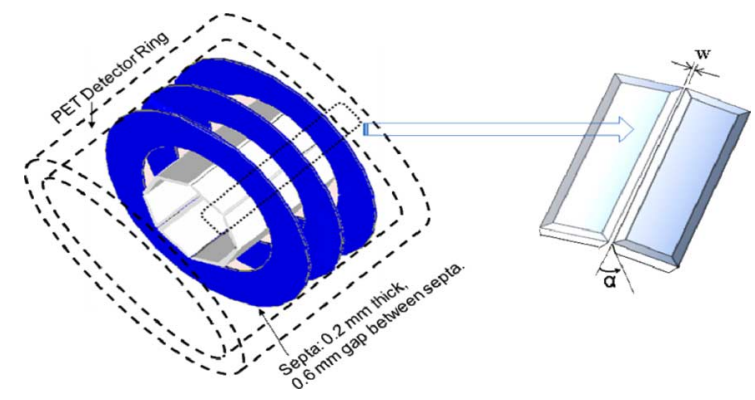

Fig. 1. This diagram on the left illustrates the concept of placing a collimator insert inside a PET detector ring for SPECT imaging. The collimator consists of multiple slits and annular-shaped septa. The slit-aperture with open angle $\alpha$ and gap width $w$ is shown on the right.

performance over the entire axial FOV and the simplicity of acquisition orbit required for tomographic imaging with this collimator. As shown in Fig. 1, the slit-slat collimator consists of knife-edged lead plates that form slits and annular septa perpendicular to the slits. The setup provides a 2D fan-beam like collimation. Tomographic acquisition can be carried out by either rotating the collimator or the imaging object. To take advantage of the strong system and noise modeling capability of iterative statistical image reconstruction methods, we chose the ordered-subsets expectation-maximization (OSEM) algorithm [4] as the primary means of image reconstruction.

The accuracy of system matrix used in image reconstruction greatly affects the performance of iterative statistical reconstruction methods, as demonstrated by several groups [5]-[8]. In addition to the accuracy consideration, we intended to develop a method that is flexible and therefore can be used for different system configurations. This is very useful at the system design and development stage when the actual system is not developed and different concepts need to be evaluated. It is also highly desired that the method is fast and therefore can generate a new system matrix in a short period of time to accommodate the possible configuration changes, e.g. mechanical changes introduced by moving the collimator in and out of the PET gantry on the developed system.

Representative SPECT system matrix modeling approaches proposed by others include analytical calculation [9]-[12], Monte Carlo simulation [13]-[15], and experimental data [16], [17]. Ultimately, measuring the point spread functions by scanning a point source throughout the field-of-view (FOV) on the actual system can be the most accurate way to obtain the system matrix. However, this approach is constrained by : 1) the availability of an actual system;2) the availability of a precise point source placement mechanism; and 3) the long scan time to acquire high-statistical data at all the grid points 
of the FOV. These constraints are prohibitive for a system in the development stage, in which case the actual scanner is either not existing physically or is frequently changed for performance optimization. Analytical and Monte Carlo modeling can be carried out on virtual scanners and are more suitable for new system investigation. While analytic methods are based on parameterized scanners and may suffer the consequence of over-simplifying photon transportation and detection processes on the actual collimator and scanner, a Monte Carlo simulation based method can incorporate more detailed realistic collimator and scanner settings in the modeling process. The recent advances in computer technology and Monte Carlo simulation software [18] also greatly facilitate the application of Monte Carlo approach. An additional and significant advantage of Monte Carlo modeling is that it is able to characterize individual physical factors in the virtual scanner environment, so the system response for different classes of events can be derived [19]. This is also valuable for implementing scatter correction techniques. A drawback of Monte Carlo simulation for system matrix modeling is the intensive computation. Considering that the system matrices of modern scanners is typically large and minimal noise (high statistics) is preferred, the simulation time can be too long to be practically useful without acceleration techniques.

In this paper, the system matrix derivation method and techniques that we designed and evaluated for the slit-slat collimator-based animal SPECT system are presented. As compared to previous published work using a similar approach, the primary contributions in this work are 1) the study of the slitslat collimator's axial symmetry; and 2) the detailed techniques of validating the system matrix with a measured point source and phantom data. As one building block for developing a new system, this work focused on qualitative imaging. Quantitative imaging techniques such as attenuation and scatter corrections have not been implemented on the prototype system and therefore are not discussed in this paper.

\section{MATERIALS AND METHODS}

\section{A. Animal PET Scanner and Collimator}

The animal PET scanner used in this study was a microPET Focus-120 system (Siemens Preclinical Solutions, Knoxville, $\mathrm{TN})$. It consists of 4 detector rings with $14.7 \mathrm{~cm}$ diameter and $7.6 \mathrm{~cm}$ axial extent [20]. Each detector ring has $24 \mathrm{de}-$ tector blocks. Each block consists of a $12 \times 12$ array of $1.51 \times 1.51 \times 10 \mathrm{~mm}^{3}$ Lutetium Oxyorthosilicate (LSO) crystals. The average energy resolution of the detector was $\sim 37 \%$ for ${ }^{57}$ Co. The energy window was set at $120-160 \mathrm{keV}$ for simulation and experiment.

The prototype slit-slat collimator used to convert the PET scanner for SPECT imaging is illustrated in Fig. 1. It consisted of 8 slits and 21 septa (only 3 septa are shown in the figure). The slit plates were $2.5-\mathrm{mm}$ thick lead. The slits were aligned to the axial direction of the PET scanner. The annular septa were $0.25-\mathrm{mm}$ thick tungsten, and were spaced evenly $(0.55 \mathrm{~mm}$ apart) along the axial direction outside the slit plates. The slit open-angle $\alpha$ and aperture-width $w$ were 45 degree and $0.6 \mathrm{~mm}$, respectively. The centers of the septa were aligned to the axial axis of the microPET scanner. The inside and outside diameters of the septa were 84 and $132 \mathrm{~mm}$, respectively. In the preliminary studies reported in this work, the imaging objects were rotated around the axial axis of the PET scanner for tomographic data acquisition. The design considerations and performance evaluations of the collimator-insert SPECT system are described in a previously published work [3].

\section{B. Animal SPECT System Calibration}

The center of rotation (COR) of the imaging object and the precise locations of the slit apertures are critical parameters for accurately modeling the system matrix. In addition to mechanically securing the formation of the collimator and the consistent placement of it in the scanner gantry, we implemented a method to determine accurately the COR and slit locations from two separate rotational point source scans [21]. The method is an extension of the published three-point-source approach for a single pinhole collimator [22]. The accuracy of the system geometric parameters was verified by imaging a point source in SPECT mode. The reconstructed point source image was artifact free with the COR and slit location values obtained [21].

\section{Theory of Deriving System Matrix From Monte Carlo Simulation}

The relationship between an emission source, $\theta$, and the detected events, $Y$, can be explicitly summarized as

$$
E\left(Y_{i}\right)=L \sum_{j=1}^{J} A_{i j} \cdot N_{i} \cdot c_{i j} \cdot \theta_{j}+S_{i}
$$

where $i$ is a detector-crystal index, $E()$ means the estimate, so $E\left(Y_{i}\right)$ is the estimated detected events in crystal $i . L$ is the dimensionless product of the decay factor, the live time factor, and the gamma emission branching fraction; $N_{i}$ accounts for the sensitivity of crystal $i, \theta_{j}$ is the emission activity at image voxel $j, A_{i j}$ represents the attenuation factor for the emitted photons from $j$ to reach $i, c_{i j}$ is the probability of emissions from voxel $j$ being detected by crystal $i, S_{i}$ is the object scattered events detected in crystal $i$. The focus of this paper is to obtain the $c_{i j}$ for all the crystals and image voxel, i.e., to obtain the system matrix $C$.

In a Monte Carlo simulation study, the origin of a detected event can be exactly identified. Let $Y_{i K}$ be a large enough number of events originated from $K$ and detected in $i$, and assume the source is distributed in vacuum, it can be derived from (1) that

$$
c_{i K}=\frac{Y_{i K}}{D},
$$

where $D$ is a leading constant that collapsing the decay factor $L$, attenuation factor $A$, activity concentration $\theta$, and crystal sensitivity. With a uniform source distributed in vacuum covering the FOV, apply (2) for all the crystals $i$ and voxels $K$, the system matrix $C$ can be obtained.

\section{Nonzero Elements of the System Matrix}

The number of elements in the system matrix in its direct form is the product of $I$ and $J$, where $I$ and $J$ are the total number of crystals and image voxels, respectively. For high resolution 


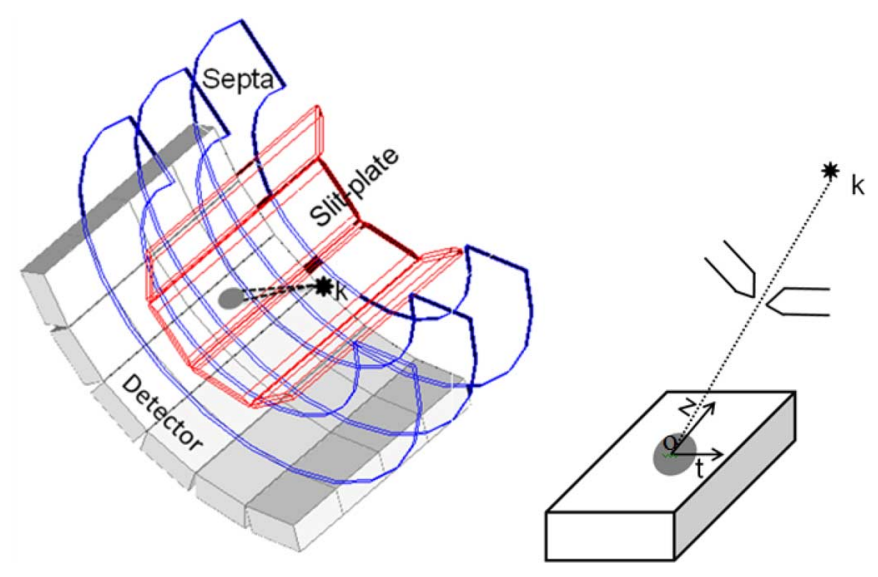

Fig. 2. The diagram on the left illustrates a point source at $\mathrm{K}$ projects through one slit opening only to a small area of crystals on the detector ring. The diagram on the right shows the coordinate-system's orientation and variables used for storing the submatrix for the source at $\mathrm{K}$.

imaging systems, the system matrix size can be very large and this can pose difficulty in file storage and computing memory. For example, the animal PET system used in this work has 13824 crystals. i.e., $I=13824$. For a FOV of $4 \times 4 \times 7.6 \mathrm{~cm}^{3}$, which is expected for a complete collimator insert with the current design, $J$ is $100 \times 100 \times 190=1.9 \times 10^{6}$ if a cubic voxel of $(0.4 \mathrm{~mm})^{3}$ is used. In this case, the system matrix has $2.6 \times 10^{10}$ elements or $\sim 100 \mathrm{~GB}$ if a 4-byte float is used for each element's value. This size is too cumbersome for file storage and difficult to load into computer memory. It is necessary to use the sparsity of the system matrix to reduce its size.

The slit-slat collimator confines an emission voxel's exposure to a small fraction of the total detector crystals. These crystals correspond to the nonzero elements of the system matrix for the emission voxel. Fig. 2 illustrates an emission voxel $K$ and its exposure on the detector surface. The photons from $K$ project to a small active area on a detector block through one slit and the septa. Let $L_{z}$ and $L_{t}$ be the number of crystals of the source projection area in the axial and tangential directions, respectively, then the number of crystals that detect photons from $K$ is $L_{z} \cdot L_{t} \cdot N_{\text {slit }}$, the rest of the crystals do not detect any events from $K$, where $N_{s l i t}$ is the number of slits. The $L_{z} \cdot L_{t} \cdot N_{\text {slit }}$ crystals are collectively noted as $P$.

To reduce the system matrix size, we choose to save only the $c_{i K, i \in P}$ elements for each voxel $K$. For each slit, $c_{i K, i \in P}$ is the point spread function of $K$, which is a 2-D function as shown in Fig. 2. Each 2-D function can be recorded in a table with a reference point $O$, which corresponds to the crystal upon which the gamma rays from source $K$ shine after passing through the center of the slit opening. Assume $t$ and $z$ are the coordinates in the tangential and axial directions relative to the origin respectively, as shown in Fig. 2, for each slit, $c_{i K, i \in P} \approx f(t, z)$ is the nonzero subsystem matrix, or effective point spread function (PSF), that needs to be recorded. For 8 slits, there are 8 such areas.

Tomographic imaging on our prototype system is achieved through rotating the image object and acquiring data with multiple step-shot acquisitions. The slit-slat collimator stays still.

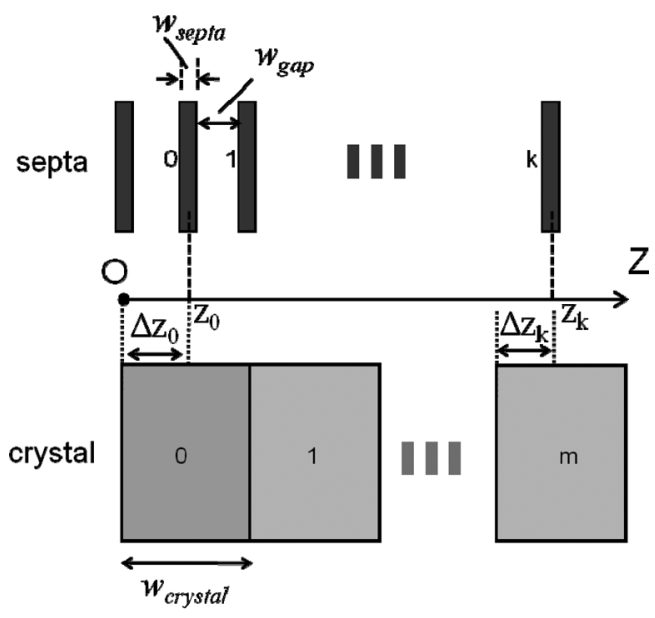

Fig. 3. The relative position of septa to a crystal, $\Delta z$, is the distance between the center-line passing through the middle of a septa and one edge of a crystal.

First, a system matrix for the image volume at its start position is derived. For the image object at a rotated angle, we rotate the image volume with the same angle and use the same system matrix for image reconstruction with necessary 2-D interpolation in the trans-axial plane.

\section{E. Base System Matrix in Axial Direction}

The 2-D collimation formed by the slit-slat collimator indicates that the system response has axial symmetries. The base axial system matrix, if identified, can be used to further reduce the image volume needed for modeling the system matrix.

For an ideal detector with continuous crystal in the axial direction, the PSF for a point source is only dependent on the position of the point source relative to the septa. The PSF that moves along the axial direction will repeat itself in cycles, and the length of the cycle is the repetition period of the septa. With the involvement of the crystal array, as shown in Fig. 3, the relative position of the septa to crystal is an additional factor contributing to the PSF. To identify and characterize the axial symmetry, we look for the cycle in terms of the relative position of the septa to the crystal. As illustrated in Fig. 3, assuming that relative position of the 1st (index 0) septa to 1st (index 0) crystal is $z_{0}$, the relative position of $k^{t h}$ septa to $m^{t h}$ crystal is $z_{k}$, which is

$$
z_{k}=\Delta z_{0}+k \cdot\left(w_{\text {septa }}+w_{\text {gap }}\right)=\Delta z_{k}+m \cdot w_{\text {crystal }}
$$

where $w_{\text {septa }}, w_{\text {gap }}$, and $w_{\text {crystal }}$ are the thickness of septa, the septa gap and crystal width, respectively; $k$ and $m$ are septa and crystal indices, both starting from 0 .

Assuming that at the $k^{\text {th }}$ septa, the relative position of the septa starts to repeat its relative position to a crystal facing it, that is $\Delta z_{0}=\Delta z_{k}$, then $k$ and $m$ are the minimal integers that solves the (5), i.e.

$$
k \cdot\left(w_{\text {septa }}+w_{\text {gap }}\right)=m \cdot w_{\text {crystal }} .
$$

The cycle of PSF in axial direction is $k \cdot\left(w_{\text {septa }}+w_{\text {gap }}\right)$ or $m \cdot w_{\text {crystal }}$. With the prototype system reported in this work, 
$w_{\text {septa }}=0.25 \mathrm{~mm}, w_{\text {gap }}=0.55 \mathrm{~mm}$, and $w_{\text {crystal }}=1.6 \mathrm{~mm}$, replace the values in the $(5)$,

$$
k=2 m \text {. }
$$

The smallest integers of $k$ and $m$ that solve (5) are 2 and 1 , respectively. So the cycle length, or the axial symmetry base length is $1.6 \mathrm{~mm}$. To account for possible manufacturing inaccuracies, we used $3.2 \mathrm{~mm}$ as the axial base length in our system matrix derivation.

\section{F. Simulation for System Matrix Derivation}

The Monte Carlo simulation package GATE 3.0.0 [23] was used for emulating the virtual scanner with the exact collimator and detector configuration. Simulation settings were made to ensure the highest resemblance of the virtual scanner and the real system. The simulation tasks were distributed to multiple computer nodes on a Linux cluster with random seeds [24] pregenerated and saved in a file. The simulation results generated on multiple computer nodes were collected and analyzed. The system matrix of an emission system can be derived directly according to (2).

The phantom used for generating the system matrix derivation data was a 3.2-cm diameter, 3.2-cm-long cylinder filled with uniform $99 \mathrm{~m}$ Tc source in air. The cylinder was placed in the center of the FOV of the SPECT system.

\section{G. Phantom Studies}

1) Point Source Measurements for System Matrix Validation: The quality of the system matrix derived from the Monte Carlo simulation was assessed with PSFs obtained from a point source measurement. On one side, we acquired the projection data of a sealed ${ }^{57}$ Co point source (Isotope Products Laboratories, Burbank, CA) at a know position, as shown in Fig. 2. The size of the ${ }^{57}$ Co source was $1 \mathrm{~mm}$ in diameter and $1 \mathrm{~mm}$ in length. On another side, a digital point source with the same size was placed at the same location in the image space and forward projected with the system matrix derived to generate the counterpart PSFs through the slits. The PSFs obtained from both sides were then compared in terms of the projection image pattern and width in terms of FWHM. The ${ }^{57} \mathrm{Co}$ was used for this validation to avoid a liquid point source preparation and to ensure consistency in terms of source size and activity.

2) Validating the Axial Symmetry: The ${ }^{57}$ Co point source was put at the $5 \mathrm{~mm} 6$ o'clock position in relation to the COR, and scanned at 11 positions over a $3.2 \mathrm{~mm}$ range in the axial direction, as illustrated in Fig. 4. Initially, the study was also designed for evaluating the axial resolution of the system so variable source intervals would allow individual point source measurement to be combined to form point-source patterns with different source-to-source distances. The axial resolution study was later accomplished using the modified Defrise phantom, as described in Section II-G-3).

The projection profiles of the point source at each axial location were then evaluated in terms of FWHM for evaluating the cyclic pattern as predicted in Section II-E.

3) Tomographic Phantom Studies: An ultramicro hot-rod phantom (Data Spectrum Inc., Hillsborough, NC) filled with

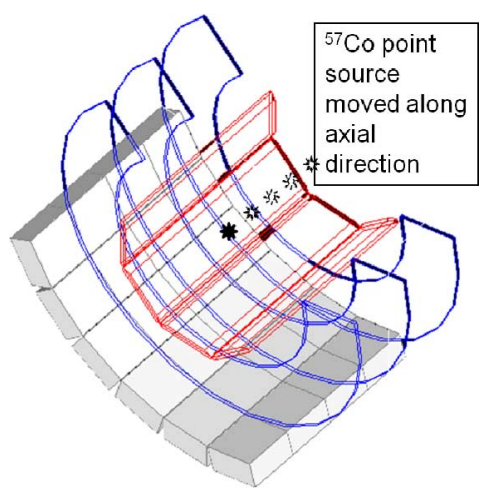

Fig. 4. The ${ }^{57} \mathrm{Co}$ point source was scanned at 11 positions over $3.2 \mathrm{~mm}$ range in axial direction to validate the axial symmetry.

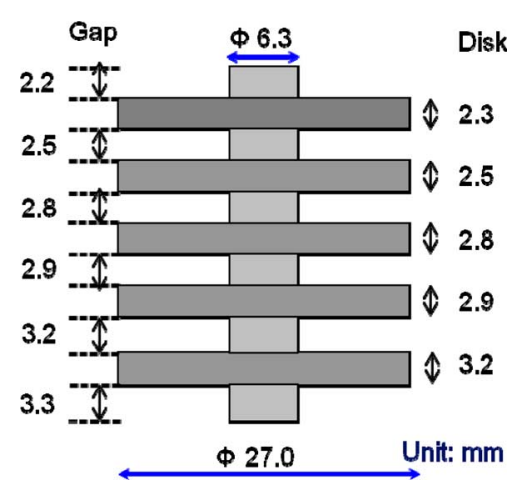

Fig. 5. The drawing shows the cross section view of a modified Defrise phantom insert designed for evaluating axial spatial resolution. The thicknesses of the plexiglass discs (in gray) and the gaps between discs, both labeled in the drawing, were nonuniform and incremental from one end to the other end of the insert.

$\sim 1 \mathrm{mCi} 99 \mathrm{mTc}$ was scanned for assessing the resolution performance of the reconstruction in the transverse plane. It was rotated for 120 steps at 3 degree/step, and scanned for 128 seconds at each step.

To assess the axial resolution performance of the derived system matrix in image reconstruction, we used a modified Defrise phantom insert (Fig. 5). The insert was put in a cylinder and the cylinder was filled with $7.2 \mathrm{mCi}$ of $99 \mathrm{mTc}$. Due to the limited axial FOV $(1.6 \mathrm{~cm})$ of the prototype system, the phantom was scanned at two axial positions, which were $1.6 \mathrm{~cm}$ apart. The images were reconstructed separately and stitched together.

The activity and acquisition parameters for this study were chosen to provide adequate statistics and sampling to evaluate the resolution performance of the reconstruction implementation. Practical dose and acquisition protocol studies are beyond the scope of this manuscript.

\section{H. Data Processing and Image Reconstruction}

The intrinsic radioactivity of ${ }^{176} \mathrm{Lu}$ in the lutetium oxyorthosilicate (LSO) crystals was estimated based on a premeasured long LSO background scan and subtracted [25] from the measured projections. The energy window used for LSO background measurement matched that of the to-be corrected data. The sensitivity coefficients of the crystals were obtained by scanning a uniform cylinder phantom filled with a $99 \mathrm{~m} \mathrm{Tc}$ 


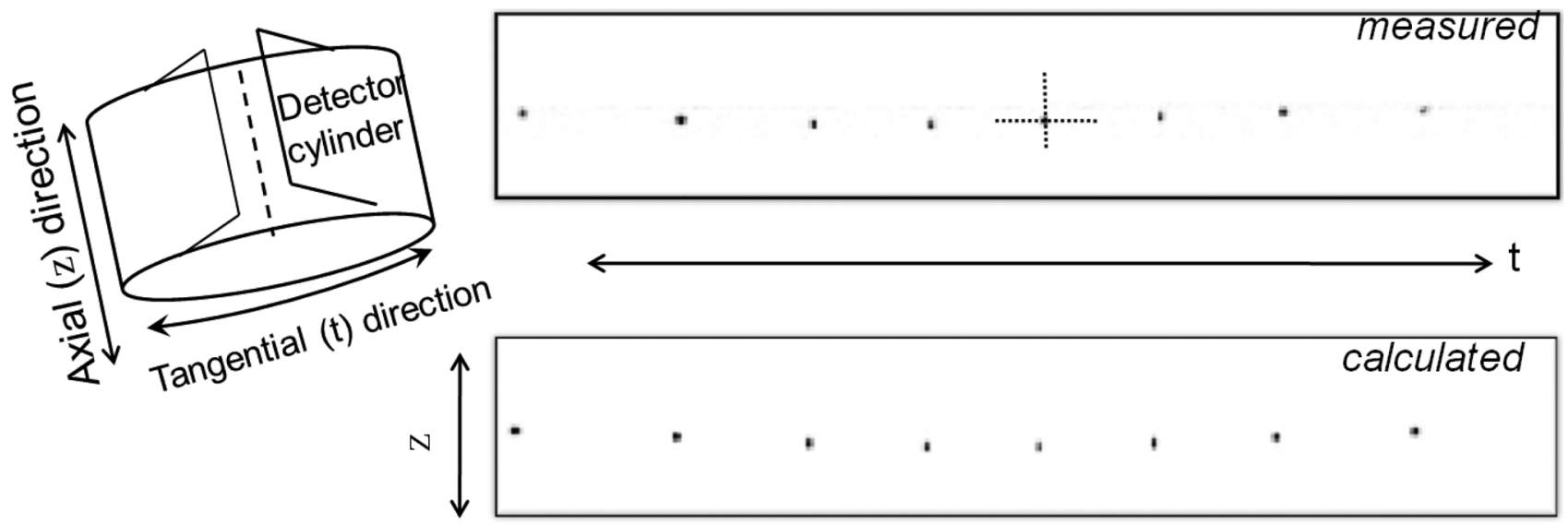

Fig. 6. The drawing on the left illustrates the detector ring in its cylindrical shape and that the images shown on the right were from opening and unfolding the detector ring. The two images on the right compares the point source projection image from a measurement (top row) and from forward projecting the point source with system matrix derived (bottom row). The dashed arrows in the top image shows the orientations of the section profiles analyzed in Figs. 7 and 8.

solution with collimator removed and by using the ${ }^{57}$ Co blank scan designed for PET system's attenuation correction [26] for ${ }^{99} \mathrm{~m} \mathrm{Tc}$ and ${ }^{57} \mathrm{Co}$ studies, respectively. The normalization coefficients were applied to the projection data precorrected for LSO background and radioactive decay before image reconstruction. Due to the small size of the targeted imaging objects, i.e., rats or mice, with this system, scatter and attenuation were considered to have limited effects and not implemented in this work.

An iterative reconstruction program with ordered subset expectation maximization (OSEM) algorithm was used for all the image reconstruction tasks. The number of iterations and subsets, 10 and 8 respectively, were empirically determined to give the optimal results. The reconstructed image voxel size was $0.4 \mathrm{~mm} \times 0.4 \mathrm{~mm} \times 0.4 \mathrm{~mm}$.

\section{RESULTS}

\section{A. System Matrix Size}

The nonzero elements in a PSF of the SPECT system can be adequately included within 8 (tangential direction) by 4 (axial direction) crystals. So the size of one emission voxel's PSF is $L_{z} \times L_{t} \times N_{\text {slit }}=8 \times 4 \times 8=256$. This is the size of the system matrix to be stored for the voxel. For a transaxial slice with FOV of $4 \mathrm{~cm} \times 4 \mathrm{~cm}$ and base axial length of $3.2 \mathrm{~mm}$, and with a voxel size of $0.4 \times 0.4 \times 0.4 \mathrm{~mm}^{3}$, the number of voxels in the system matrix is $N_{x} \times N_{y} \times N_{z}=100 \times 100 \times 8$, where $N_{x}, N_{y}$, and $N_{z}$ are the number of voxels along $\mathrm{x}, \mathrm{y}$, and $\mathrm{z}$ directions for the image volume modeled in the system matrix. If a 4-byte float is used for each matrix element, the total system matrix size is $256 \times 8 \times 104 \times 4=80 \mathrm{MB}$.

\section{B. Simulation Time and Statistics Consideration}

A Monte Carlo simulation that generated $\sim 6.3 \times 10^{8}$ counts for the system matrix derivation took a total of 22500 processorhours on a 1000-node Linux cluster, each node has dual 3.2-GHz Intel Xeon processors and a 4-GB RAM. This is equivalent to about $2.5 \mathrm{~d}$ computing time with about 400 processors allocated for this task.
With the parameters described in Section III-A for the system matrix derivation, in each PSF, the average number of elements with more than $5 \%$ of the maximum count is $\leq 10$. So the total number of PSF elements with nonnegligible amplitude is about $N_{x} \times N_{y} \times N_{z} \times 10=8 \times 10^{5}$, the average counts in one such element is about 800 , the statistical error of element is $\sim 3.5 \%$ and is considered to be low enough for iterative reconstruction use.

\section{Validation of the Derived System Matrix With Measured $P S F$}

A measured projection image, i.e., the direct projection count distribution map over the detector array, of the ${ }^{57} \mathrm{Co}$ point source is compared to the point source's system matrix derived projection image in Fig. 6. As a result of using 8 slit-apertures in the collimator insert, the point source has 8 projection locations in each image. In other words, Fig. 6 shows the comparison of measured and calculated PSFs. The septa of the collimator-insert were slightly tilted due to mechanical assembly inaccuracies. As a result, the point source's projections were not on a horizontal line. The tilt and off-centered position of the point source introduced the observed intensity variations in PSF. The PSF locations in the two images matched well, indicating that the geometric parameters used in the system matrix derivation and projection image generation were accurate. Although the projection patterns of the point source in the two images resemble each other in general, they are not exactly the same when inspected closely.

The section profiles in the axial and tangential directions of the measured and calculated PSF are compared in Fig. 7 in terms of FWHM, which was obtained with Gaussian fitting. For the 8 PSFs from 8 slits, the average differences in terms of FWHM are about 0.4 and $0.5 \mathrm{~mm}$ in the tangential and the axial directions, respectively. The measured projections are mostly wider than that of the system matrix derived projections. This is possibly due to that underestimated slit-opening and septa gap which were used in the simulation setup for deriving the system matrix. 

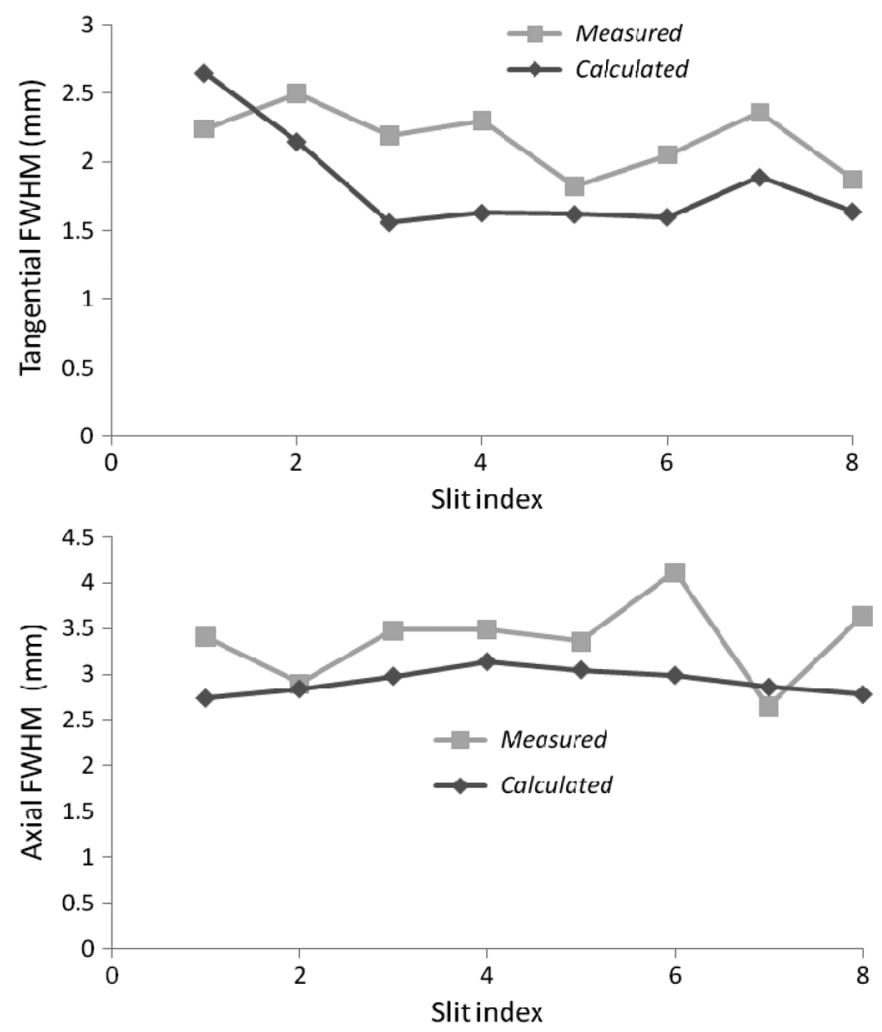

Fig. 7. A comparison of the projection profiles of the ${ }^{57} \mathrm{Co}$ point source in the images obtained from acquiring the projection data of the ${ }^{57} \mathrm{Co}$ point source (Measured), and from forward-projecting the corresponding modeled point source with the system matrix derived (Calculated). The 8 data points are the projections from 8 slit-apertures with one point source measurement. The FWHM was obtained from fitting the profiles in tangential (top) and axial (bottom) directions with a Gaussian function.

The cyclic pattern in the axial direction was confirmed by both the measured (top graph, Fig. 8) and the calculated PSFs (bottom graph, Fig. 8). The cycle periods were about 3.2 and $1.6 \mathrm{~mm}$, respectively for the measured and calculated PSFs. Given the geometrical parameters used in the simulation, the $1.6-\mathrm{mm}$ period is expected, as described in Section II-E. On the other hand, the $3.2-\mathrm{mm}$ period indicates that the actual dimensions of the crystal and septa in the prototype system were different from the values we assumed in the simulation and modeling. Consistent with what is observed in Fig. 7, the FWHM of the measured PSFs is about $0.5 \mathrm{~mm}$ wider than that of the calculated on average.

\section{Tomographic Images}

The most important test for the derived system matrix is the quality of the reconstructed images. The transverse plane image of a hot-rod phantom and the section profile passing through several hot-rods are shown in Fig. 9. The images of the 1.7 $\mathrm{mm}$ diameter hot-rods are clearly identified. There is no evident image artifact.

Fig. 10 shows a "coronal" view of the modified Defrise phantom image. The profiles show clear separation of the hot strips with $2.5 \mathrm{~mm}$ thickness. The profile shown in the lower part of Fig. 10 indicates the axial image resolution achieved with the reconstruction is $2.5 \mathrm{~mm}$ or better.
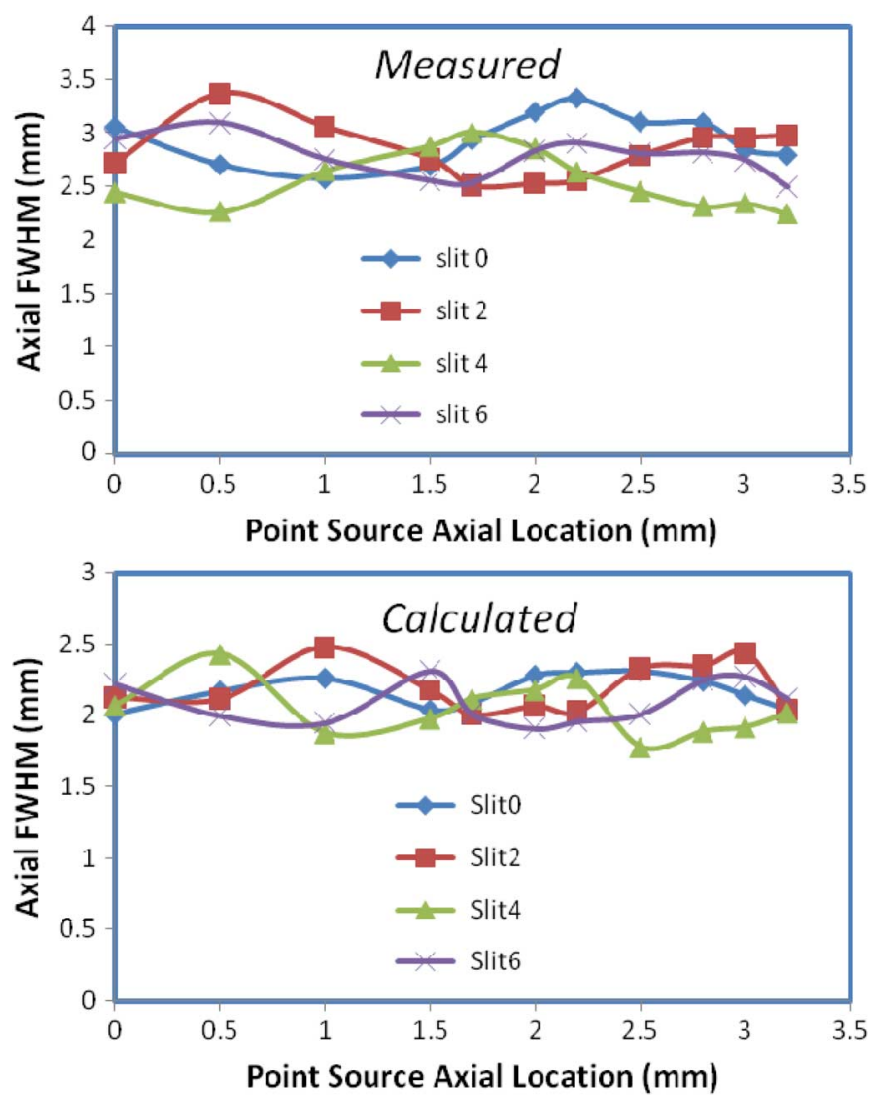

Fig. 8. The FWHM of a ${ }^{57}$ Co point source projection profile in axial direction. The ${ }^{57} \mathrm{Co}$ point source was measured at 11 axial positions over a $3.2 \mathrm{~mm}$ range. The 4 curves were from projections corresponding to 4 slit-apertures. The cyclic trend can be observed. The curves from the 4 other slit-apertures (not shown) have the same trend.

\section{DISCUSSIONS}

\section{A. Accuracy of the Simulated System Matrix}

The accuracy of the simulated system matrix is dictated by the resemblance of the simulated to the actual imaging system. The degree of resemblance is determined by the accuracy of the geometrical parameters and specifications defining the simulated system. In this work, a few geometry parameters that have dominating effects on the system matrix derivation and reconstructed image quality, i.e., the slit-aperture location and COR, were obtained through a calibration protocol with accuracy of less than $0.2 \mathrm{~mm}$ [21]. There are several other geometry parameters such as the width of slit aperture ( $w$ in Fig. 1), slit open-angle ( $\alpha$ in Fig. 1), the gap between septa ( $w_{\text {gap }}$ in Fig. 3), and septa to crystal relative position ( $\Delta z_{0}$ in Fig. 3), however, have not been included in the calibration. The accuracy for these geometrical parameters was only as good as our laboratory's mechanical assembly capability. The estimated assembly accuracy was about $0.5 \mathrm{~mm}$.

Figs. 7 and 8 show the measured FWHM value in both axial and tangential directions to be larger than the calculated PSF. This result indicates that the width of slit aperture and the gap between septa used in the simulation might be lower than the actual values of the collimator-insert. Besides, the shift between the calculated and measured cyclic pattern in Fig. 8 demonstrates that the septa to crystal relative position in the simulated 

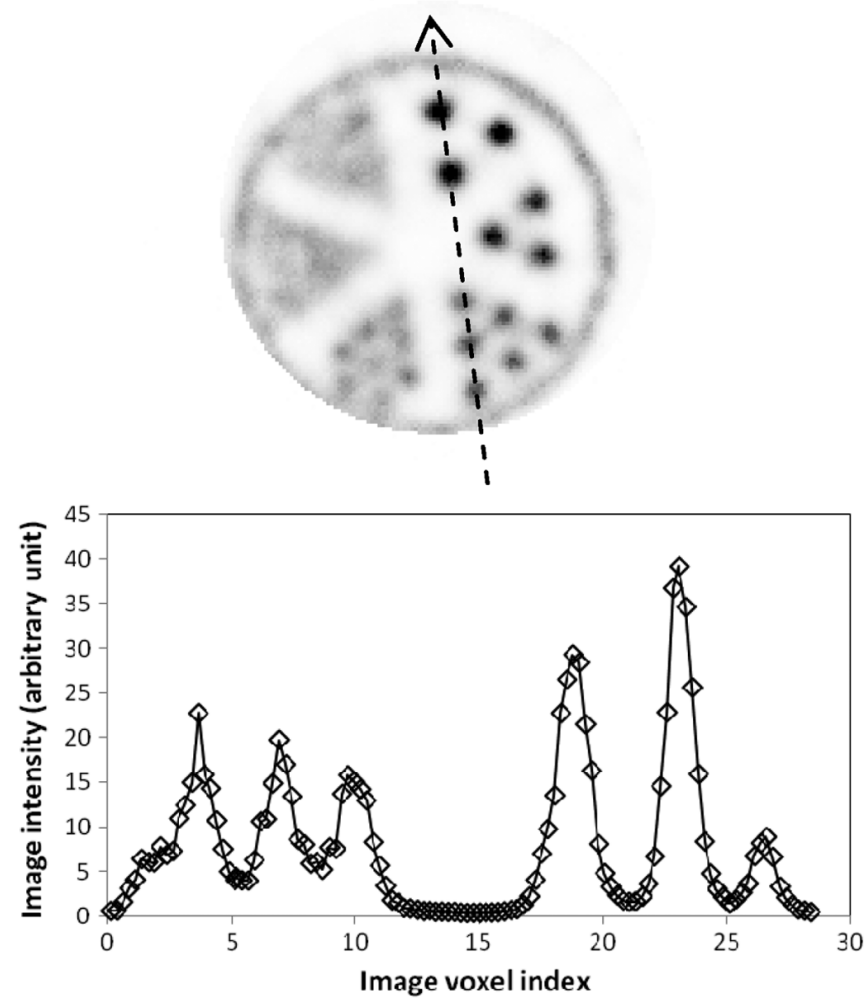

Fig. 9. The image of a transverse plane of the hot-rod phantom is shown on top. The section profile as indicated by the dashed line is shown in the bottom graph. The hot-rod diameters in the six sections were $2.4,2.0,1.7,1.35,1.0$, and $0.75 \mathrm{~mm}$, respectively. The distance between hot-rods in each section was twice that of the hot-rod diameter in the section.
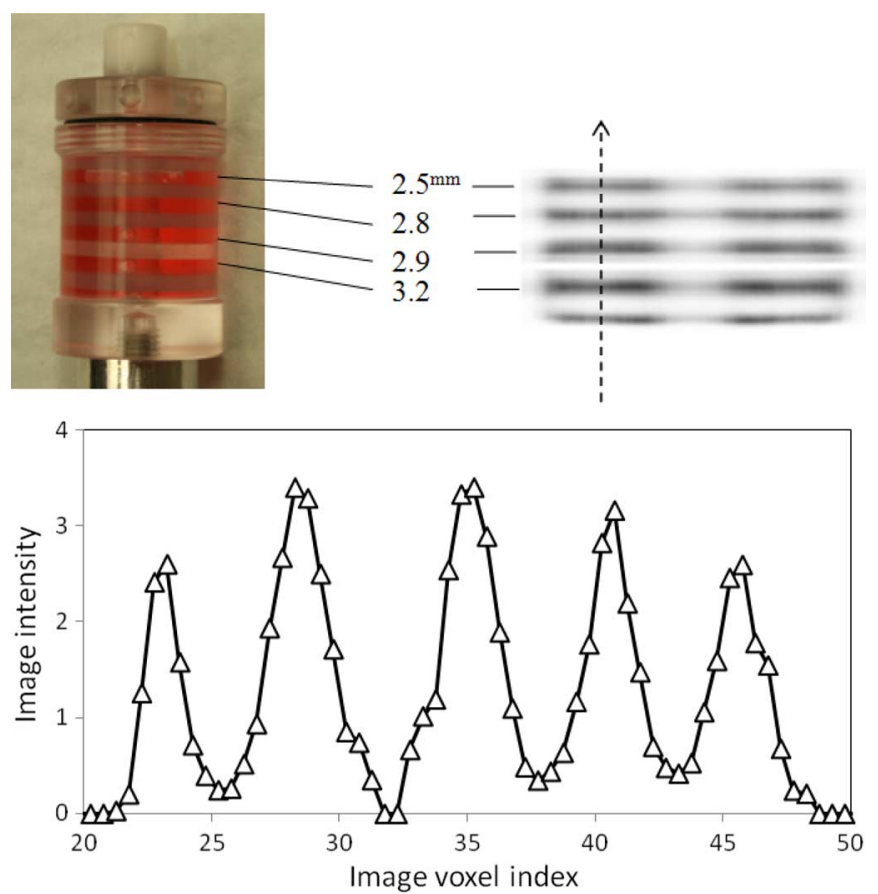

Fig. 10. A photo of the modified Defrise phantom filled with $99 \mathrm{mTc}$ activity (in red) is shown on the top-left. The image on the top-right is the "coronal" sectional view of the reconstructed image. The image profile shown in the bottom graph is from the location indicated by the dashed line overlaid on the image in the center.

and actual system were misaligned. To achieve a better match between the measured and calculated PSFs, i.e., to derive a more accurate system matrix, all the geometrical parameters identified above should be included in the calibration. Knowing the conventional geometrical parameter calibration, as the one we implement, used only the centroids of orbiting point source trajectories [27], calibrating these additional parameters may require the characterization of point source trajectory's shape. The PSF validation approach described in this work would be an important step in assessing the accuracy of the calibration.

\section{B. Acceleration of System Matrix Derivation Through Controlled Source Emission Directions}

Even with a powerful computer cluster with hundreds of nodes, it still takes a few days to generate the simulation data for deriving the system matrix for each new configuration. For quick production of system matrix using the Monte Carlo simulation data, a possible solution is to use a series of rod sources instead of the uniform cylinder source. For each rod, the axial and transverse opening angles towards a slit can be precalculated. The emission directions of the sources can then be restricted to these openings to maximize the probability of photon detection and therefore improve the simulation efficiency and reduce the simulation time.

\section{CONCLUSION}

We implemented a system matrix derivation method for an animal SPECT system using a slit-slat collimator and a PET detector system. The slit-slat collimator's axial symmetry was studied and validated through a study of measured PSFs from moving a point source over a $3.2 \mathrm{~mm}$ range of axial positions. The system matrix was first assessed with measured PSFs and then with experimental phantom studies. On average the FWHM of measured PSFs was about $0.5 \mathrm{~mm}$ wider than that of PSFs calculated from simulated system matrix. This indicated that certain geometrical parameters such as the width of slit aperture and septa gap need to be more accurately determined. The reconstructed phantom images showed that the hot-rods with 1.7 $\mathrm{mm}$ diameter in transverse plane and $2.5 \mathrm{~mm}$ hot-cold strips in coronal view could be distinguished clearly, indicating that certain degree of image resolution recovery has been achieved, and the system matrix derived was correct.

\section{ACKNOWLEDGMENT}

The authors would like to thank Dr. S. Siegel of Siemens Preclinical Solutions for valuable assistance and discussions on data acquisition, Central Radiopharmaceutical Service at SUNY Buffalo for providing radio pharmaceuticals for this study, and E. S. Crawford for editorial improvement of the paper. This work utilized the high-performance computational capabilities of the Linux Cluster, Center for Computational Research, State University of New York at Buffalo.

\section{REFERENCES}

[1] S. D. Metzler, R. Accorsi, J. R. Novak, A. S. Ayan, and R. J. Jaszczak, "On-axis sensitivity and resolution of a slit-slat collimator," J. Nucl. Med., vol. 47, pp. 1884-1890, Nov. 2006.

[2] S. Walrand, F. Jamar, M. de Jong, and S. Pauwels, "Evaluation of novel whole-body high-resolution rodent SPECT (Linoview) based on direct acquisition of linogram projections," J. Nucl. Med., vol. 46, pp. 1872-1880, Nov. 2005. 
[3] Y. Shao, R. Yao, T. Ma, and P. Manchiraju, "Initial studies of PETSPECT dual-tracer imaging," in Proc. IEEE Nucl. Sci. Symp. Med. Imag. Conf. Rec., 2007, pp. 4198-4204.

[4] H. M. Hudson and R. S. Larkin, "Accelerated image reconstruction using ordered subsets of projection data," IEEE Trans. Med. Imag., vol. 13, pp. 601-609, 1994.

[5] I. Laurette, G. L. Zeng, A. Welch, P. E. Christian, and G. T. Gullberg, "A three-dimensional ray-driven attenuation, scatter and geometric response correction technique for SPECT in inhomogeneous media," Phys. Med. Biol., vol. 45, pp. 3459-3480, Nov. 2000.

[6] B. M. W. Tsui, E. C. Frey, X. Zhao, D. S. Lalush, R. E. Johnston, and W. H. Mccartney, "The importance and implementation of accurate 3D compensation methods for quantitative SPECT," Phys. Med. Biol., vol. 39, pp. 509-530, Mar. 1994.

[7] J. Y. Qi, R. M. Leahy, S. R. Cherry, A. Chatziioannou, and T. H. Farquhar, "High-resolution 3D Bayesian image reconstruction using the microPET small-animal scanner," Phys. Med. Biol., vol. 43, pp. 1001-1013, Apr. 1998.

[8] J. Qi and R. H. Huesman, "Effect of errors in the system matrix on maximum a posteriori image reconstruction," Phys. Med. Biol., vol. 50, pp. 3297-312, Jul. 21, 2005.

[9] M. F. Smith and R. J. Jaszczak, "The effect of gamma ray penetration on angle-dependent sensitivity for pinhole collimation in nuclear medicine," Med. Phys., vol. 24, pp. 1701-1709, Nov. 1997.

[10] S. D. Metzler, J. E. Bowsher, M. F. Smith, and R. J. Jaszczak, “Analytic determination of pinhole collimator sensitivity with penetration," IEEE Trans. Med. Imag., vol. 20, pp. 730-741, Aug. 2001.

[11] S. D. Metzler, J. E. Bowsher, K. L. Greer, and R. J. Jaszczak, "Analytic determination of the pinhole collimator's point-spread function and RMS resolution with penetration," IEEE Trans. Med. Imag., vol. 21, pp. 878-887, Aug. 2002.

[12] M. F. Smith and R. J. Jaszczak, "An analytic model of pinhole aperture penetration for 3D pinhole SPECT image reconstruction," Phys. Med. Biol., vol. 43, pp. 761-775, Apr. 1998.

[13] F. J. Beekman and B. Vastenhouw, "Design and simulation of a highresolution stationary SPECT system for small animals," Phys. Med. Biol., vol. 49, pp. 4579-4592, Oct. 7, 2004.

[14] N. U. Schramm, G. Ebel, U. Engeland, T. Schurrat, M. Behe, and T. M. Behr, "High-resolution SPECT using multipinhole collimation," IEEE Trans. Nucl. Sci., vol. 50, pp. 315-320, Jun. 2003.

[15] S. Vandenberghe, S. Staelens, C. L. Byrne, E. J. Soares, I. Lemahieu, and S. J. Glick, "Reconstruction of 2D PET data with Monte Carlo generated system matrix for generalized natural pixels," Phys. Med. Biol., vol. 51, pp. 3105-3125, Jun. 21, 2006.
[16] F. J. Beekman, F. van der Have, B. Vastenhouw, A. J. A. van der Linden, P. P. van Rijk, and J. P. H. Burbach et al., "U-SPECT-I: A novel system for submillimeter-resolution tomography with radiolabeled molecules in mice," J. Nucl. Med., vol. 46, pp. 1194-1200, Jul. 2005.

[17] L. R. Furenlid, D. W. Wilson, C. Yi-chun, K. Hyunki, P. J. Pictraski, and M. J. Crawford et al., "FastSPECT II: A second-generation highresolution dynamic SPECT imager," in Proc. IEEE Nucl. Sci. Symp. Conf. Rec., 2002, vol. 3, pp. 1375-1379.

[18] I. Buvat and I. Castiglioni, "Monte Carlo simulations in SPET and PET," Q. J. Nucl. Med., vol. 46, pp. 48-61, Mar. 2002.

[19] F. J. Beekman, J. M. denHarder, M. A. Viergever, and P. P. vanRijk, "SPECT scatter modelling in nonuniform attenuating objects," Phys. Med. Biol., vol. 42, pp. 1133-1142, Jun. 1997.

[20] R. Laforest, D. Longford, S. Siegel, D. F. Newport, and J. Yap, "Performance evaluation of the microPET (R)-FOCUS-F120," IEEE Trans. Nucl. Sci., vol. 54, pp. 42-49, Feb. 2007.

[21] T. Ma, R. Yao, and Y. Shao, "A SVD-based method to assess the uniqueness and accuracy of SPECT geometrical calibration," IEEE Trans. Med. Imag., 2009, to be published.

[22] D. Beque, J. Nuyts, P. Suetens, and G. Bormans, "Optimization of geometrical calibration in pinhole SPECT," IEEE Trans. Med. Imag., vol. 24, pp. 180-190, Feb. 2005.

[23] S. Jan, G. Santin, D. Strul, S. Staelens, K. Assie, and D. Autret et al., "GATE: A simulation toolkit for PET and SPECT," Phys. Med. Biol., vol. 49, pp. 4543-61, Oct. 7, 2004.

[24] L. Maigne, D. Hill, P. Calvat, V. Breton, R. Reuillon, and Y. Legre et al., "Parallelization of Monte Carlo simulations and submission to a grid environment," Parallel Process. Lett., vol. 14, pp. 177-196, 2004.

[25] R. Yao, T. Ma, and Y. Shao, "Lutetium oxyorthosilicate (LSO) intrinsic activity correction and minimal detectable target activity study for SPECT imaging with a LSO-based animal PET scanner," Phys. Med. Biol., vol. 53, pp. 4399-4415, 2008.

[26] W. Lehnert, S. R. Meikle, S. Siegel, D. Newport, R. B. Banati, and A. B. Rosenfeld, "Evaluation of transmission methodology and attenuation correction for the microPET Focus 220 animal scanner," Phys. Med. Biol., vol. 51, pp. 4003-4016, Aug. 21, 2006.

[27] F. Noo, R. Clackdoyle, C. Mennessier, T. A. White, and T. J. Roney, "Analytic method based on identification of ellipse parameters for scanner calibration in cone-beam tomography," Phys. Med. Biol., vol. 45, pp. 3489-3508, Nov. 2000. 